\title{
Protein expression of Fragile Histidine Triad and cyclooxgenase- 2 in serrated neoplasia of the colorectum
}

\author{
AKIHIRO TAMOTO, KAZUO YASHIMA, KOHEI HOSODA, SOHEI YAMAMOTO, \\ SOICHIRO KAWATA, YUICHIRO IKEBUCHI, KAZUYA MATSUMOTO, KOICHIRO KAWAGUCHI, \\ KENICHI HARADA, YOSHIKAZU MURAWAKI and HAJIME ISOMOTO
}

Division of Medicine and Clinical Science, Faculty of Medicine, Tottori University, Yonago 683-8504, Japan

Received October 12, 2016; Accepted March 9, 2017

DOI: $10.3892 / 01.2017 .6634$

\begin{abstract}
The adenoma-carcinoma sequence (ACS) and the serrated pathway are two distinct developmental routes leading to the formation of colorectal carcinoma (CRC). However, the mechanism triggered by the serrated pathway remains unclear. Therefore, to clarify the molecular and clinicopathological characteristics of the serrated tumorigenic pathway, immunohistochemistry was used to examine the expression of Fragile Histidine Triad (FHIT), cyclooxygenase-2 (COX-2), MutL homolog 1 (MLH1), MutS protein homolog 2 (MSH2) and P53 in endoscopically resected samples of 62 serrated polyps. These samples included 20 hyperplastic polyps (HPs), 16 traditional serrated adenomas (TSAs), 26 sessile serrated adenoma/polyps (SSA/Ps), 20 non-serrated adenomas, 20 carcinoma in adenomas (CIAs) and 18 early pure CRCs without any adenoma component (EPCs). FHIT expression was markedly reduced or absent in 50\% of TSA samples, $92.3 \%$ of SSA/Ps and 44\% of EPCs, but only rarely in HPs, non-serrated adenomas and CIAs. COX-2 expression was more common in non-serrated adenomas compared with in serrated polyps, and was present in 25 and $3.2 \%$ of the cases respectively $(\mathrm{P}<0.01)$. Furthermore, COX-2 expression was more frequent in CIAs (60\%) compared with in EPCs $(22.2 \%$; $\mathrm{P}<0.05)$. The incidence of negative COX-2 expression was higher in FHIT-negative $\mathrm{SSA} / \mathrm{Ps}$ compared with in FHIT-positive SSA/Ps $(\mathrm{P}=0.08)$. A total of $16.7 \%$ of EPC samples and $11.5 \%$ of SSA/Ps demonstrated a loss of MLH1/MSH2 expression, but none of the other tumor types did. P53 overexpression was significantly increased in EPC (77.8\%) and CIA (60\%) samples compared with in HP (0\%), TSA $(6.6 \%)$, SSA/P $(0 \%)$ and non-serrated adenoma $(10 \%)$ samples $(\mathrm{P}<0.01)$. These findings demonstrated that there are different expression patterns between the serrated
\end{abstract}

Correspondence to: Dr Kazuo Yashima, Division of Medicine and Clinical Science, Faculty of Medicine, Tottori University, 36-1 Nishicho, Yonago 683-8504, Japan

E-mail: yashima@med.tottori-u.ac.jp

Key words: Fragile Histidine Triad, cyclooxygenase-2, serrated pathway, sessile serrated adenoma/polyp, endoscopic resection pathway and ACS, indicating that aberrant FHIT and inhibited COX-2 expression may be associated with serrated tumorigenesis. In addition, this data indicated that EPC may contain tumors derived from the serrated pathway as well as ACS.

\section{Introduction}

Colorectal cancer (CRC) is one of the most common types of malignancy in the Western world, with a lifetime prevalence of $~ 5 \%$ (1). Despite advances in therapeutic practices, CRC remains one of the most common causes of cancer-associated mortality worldwide (1). The adenoma-carcinoma sequence (ACS) model proposes that the carcinoma arises from a pre-existing adenoma (2). The serrated pathway to CRC formation, in which serrated polyps develop into cancer, has been proposed as an alternative route to colorectal carcinogenesis $(3,4)$. According to the latest World Health Organization (WHO) classification (5), serrated polyps are categorized as hyperplastic polyps (HPs), sessile serrated adenoma-polyps (SSA/P) or traditional serrated adenomas (TSAs). The serrated pathway involves widespread aberrant DNA methylation in promoter regions, termed $\mathrm{CpG}$ island methylator phenotype (CIMP) (4). Serrated neoplasms frequently present methylation on the MutL homolog 1 (MLH1) gene, encoding a mismatch repair factor $(6,7)$. The methylation of $M L H 1$ results in a form of genomic instability termed microsatellite instability (MSI) (8). Therefore, the serrated pathway gives rise to CIMP-high carcinomas, which often also present as MSI-high characteristics (6-8).

The candidate tumor suppressor gene Fragile Histidine Triad (FHIT) resides on chromosome 3p14.2, spanning the FRA3B common fragile site (9). Abnormalities and reduced expression of FHIT are present in a variety of human cancer cell lines and primary tumors (9-12). FHIT performs a role in the development and progression of $\mathrm{CRC}(9,13)$, and its expression is associated with deficient mismatch repair (MMR) in advanced human CRCs (14-16). In addition, low FHIT protein expression is also associated with CIMP-high CRCs (17). However, relatively few studies have been published on the early serrated tumorigenic pathway. Cyclooxygenase-2 (COX-2) is also involved in colorectal carcinogenesis $(18,19)$, where its overexpression is inversely associated with MSI (20-22) and CIMP $(22,23)$. 
To the best of our knowledge, no study has comprehensively measured the expression levels of FHIT and COX-2 in serrated and non-serrated colorectal neoplasias. To clarify the molecular and clinicopathological characteristics of the serrated tumorigenic pathway, immunohistochemistry was used to analyze FHIT, COX-2, MLH1, MutS protein homolog 2 (MSH2) and P53 in 120 endoscopically-resected samples of serrated and non-serrated colorectal neoplasias, including hyperplastic polyps, SSA/Ps, TSAs, non-serrated adenomas, carcinoma in adenomas (CIAs) and early pure CRCs without any adenoma component (EPCs). Protein expression profiles were then matched with the clinicopathological characteristics of these tumors.

\section{Materials and methods}

Patient samples. Tumor specimens were obtained from 120 patients (90 males and 30 females), who had undergone endoscopic resection at Tottori University Hospital between January 2009 and December 2014. The samples included 20 HPs, 16 TSAs, 26 SSA/Ps (for a total of 62 serrated polyps), 20 non-serrated adenomas, 20 CIAs and 18 EPCs. Patients with familial adenomatous polyposis, hereditary nonpolyposis CRC and hyperplastic polyposis were excluded from the present study. Serrated lesions (HPs, SSA/Ps and TSAs) were classified on the basis of the current WHO criteria (5). The 20 HPs were subdivided into 10 microvesicular HPs (MVHPs) and 10 goblet cell HPs (GCHPs). Non-serrated adenomas measuring $>10 \mathrm{~mm}$ were used for the study. All histological types of CIAs and EPCs were well-differentiated adenocarcinomas. In addition, these neoplasms were confined to the mucosa or submucosa. Histological evaluations were performed according to the classification established by the Japanese General Rules for Clinical and Pathological Studies on Cancer of the Colon, Rectum and Anus (24). In the present study, non-serrated adenoma samples corresponded to low- or high-grade adenoma/dysplasia, and CIA and EPC samples of mucosa and submucosa corresponded to non-invasive carcinoma or intramucosal and submucosal carcinoma in the Vienna classification system (25). Tumors were divided into polypoid, and flat or depressed groups on the basis of their morphological features. Flat and depressed tumors were defined as an endoscopically visibly flat or depressed mucosal lesions with their height measuring less than half of their diameter (26). All other tumorous lesions in the colon were termed polypoid lesions. The clinical characteristics of the patients are listed in Table I. All cases were analyzed anonymously, that is, all specimens were assigned a number without any personal information. Written informed consent was obtained from all patients. The Institutional Review Board approved the present study to comply with the Declaration of Helsinki.

Immunohistochemical staining. All specimens were fixed with $10 \%$ formalin overnight at room temperature, embedded in paraffin and cut into $4 \mu \mathrm{m}$ sections. The sections were immunohistochemically stained with rabbit polyclonal anti-FHIT (dilution, 1:100; clone F130; cat. no. 18163; Immuno-Biological Laboratories Co. Ltd., Gunma, Japan), mouse monoclonal anti-COX-2 (dilution, 1:100; clone 33/Cox-2; cat. no. 610204; BD Biosciences, Franklin Lakes, NJ, USA), mouse monoclonal
anti-MSH2 (dilution, 1:100; clone FE11; cat. no. 33-7900; Oncogene Research Products; EMD Millipore, Billerica, MA, USA), mouse monoclonal anti-MLH1 (dilution, 1:50; clone G168-15; cat. no. 550838; BD Pharmingen, San Diego, CA, USA) or mouse monoclonal anti-P53 (dilution, 1:50; clone DO-7; cat. no. M7001; Dako; Agilent Technologies, Inc., Santa Clara, CA, USA) using the avidin-biotin-peroxidase complex technique. Briefly, following deparaffinization in xylene and rehydration in graded ethanol series $(100,90,80,70$ and 50\%) for $5 \mathrm{~min}$ (for each concentration) at room temperature, the sections were immersed in a citrate buffer $(0.01 \mathrm{M}, \mathrm{pH}$ 6.0) and heated in a microwave oven for 20 or $30 \mathrm{~min}$ to retrieve antigens. Endogenous peroxidase activity was subsequently blocked by incubation with $3 \% \mathrm{H}_{2} \mathrm{O}_{2}$ for $30 \mathrm{~min}$ at room temperature. Sections were then incubated with the primary antibody overnight at $4^{\circ} \mathrm{C}$. As a negative control, the primary antibody was replaced with serum Immunoglobulin $\mathrm{G}(\mathrm{IgG})$ in similar dilutions. Detection was carried out using a Vectastain Elite ABC kit (Vector Laboratories, Inc., Burlingame, CA, USA) according to the manufacturer's protocol. Diaminobenzidine was used as a chromogen, and hematoxylin was used as a counterstain. Sections were incubated with biotinylated anti-rabbit or mouse IgG and avidin-biotin-peroxidase for $30 \mathrm{~min}$ at room temperature, and visualized using diaminobenzidine tetrahydrochloride. For each specimen, $>5$ fields were viewed under a light microscope (magnification, x100) (Olympus Corporation, Tokyo, Japan). Protein expression was evaluated by two independent observers. Immunohistochemical analysis was performed in a blind manner with respect to clinical information.

Assessment of FHIT immunostaining. FHIT expression was scored on the basis of the intensity of its cytoplasmic staining. It was scored as either reduced, absent or positive, as described previously (13). Healthy colorectal epithelial cells adjacent to tumor cells exhibited a moderate to strong cytoplasmic FHIT signal from the basal portion to the luminal differentiated cells; these cells were used as positive controls.

Assessment of COX-2 immunostaining. COX-2 staining was classified as being either positive or absent. Samples with cytoplasmic staining in $\geq 30 \%$ of tumor cells were scored as having a positive signal. By contrast, samples with cytoplasmic staining in $<30 \%$ of the tumor cells were scored as having negative COX-2 expression.

Assessment of MLH1 and MSH2 immunostaining. Healthy tissue adjacent to the tumor was used as an internal positive control. MLH1 and MSH2 was localized in the nucleus in control cells. Samples with nuclear staining in $\geq 30 \%$ of tumor cells were scored as having a positive signal. By contrast, samples with a nuclear signal in $<30 \%$ of the tumor cells were categorized as having no expression (27).

Assessment of p53 immunostaining. Samples were scored as positive for $\mathrm{p} 53$ expression if they exhibited a nuclear signal and if $>30 \%$ of the tumor cells demonstrated p53 staining.

Statistical analysis. Statistical analysis was performed using the Fisher's exact test, $\chi^{2}$ test with Yates' correction, Kruskal-Wallis 
Table I. Clinicopathological features in early colorectal neoplasms.

\begin{tabular}{|c|c|c|c|c|c|c|c|}
\hline Variables & GCHP, $\mathrm{n}$ & MVHP, n & TSA, $\mathrm{n}$ & $\mathrm{SSA} / \mathrm{P}, \mathrm{n}$ & Adenoma, $\mathrm{n}$ & $\begin{array}{c}\text { Carcinoma } \\
\text { in adenoma, } \mathrm{n}\end{array}$ & $\begin{array}{l}\text { Early pure } \\
\text { carcinoma, } \mathrm{n}\end{array}$ \\
\hline Total & 7 & 13 & 16 & 26 & 20 & 20 & 18 \\
\hline Age, years $($ mean $\pm S D)$ & $61.3 \pm 15.2$ & $62.9 \pm 10.2$ & $63.6 \pm 13.5$ & $63.3 \pm 9.3$ & $70.0 \pm 11.3$ & $67.9 \pm 10.5$ & $70.7 \pm 11.7$ \\
\hline Sex (male/female) & $6 / 1$ & $10 / 3$ & $12 / 4$ & $17 / 9$ & $16 / 4$ & $16 / 4$ & $13 / 5$ \\
\hline Size, $m m($ mean \pm SD $)$ & $6.4 \pm 1.8$ & $7.3 \pm 3.5$ & $9.8 \pm 3.5$ & $9.8 \pm 4.0$ & $12.9 \pm 3.5$ & $15.5 \pm 9.9$ & $12.9 \pm 7.1$ \\
\hline Location (right/left) & $3 / 4$ & $5 / 8$ & $1 / 15$ & $14 / 12$ & $8 / 12$ & $6 / 14$ & $6 / 12$ \\
\hline $\begin{array}{l}\text { Macroscopic classification } \\
\text { (flat or depressed/polypoid) }\end{array}$ & $2 / 5$ & $7 / 6$ & $0 / 16$ & $12 / 14$ & $8 / 12$ & $9 / 11$ & $15 / 3$ \\
\hline $\begin{array}{l}\text { Depth of invasion } \\
\text { (mucosa/submucosa) }\end{array}$ & - & - & - & - & - & $14 / 6$ & $8 / 10$ \\
\hline Lymphatic invasion & - & - & - & - & - & 0 & 1 \\
\hline Venous invasion & - & - & - & - & - & 2 & 3 \\
\hline
\end{tabular}

GCHP, goblet cell hyperplastic polyps; MVHP, microvesicular hyperplastic polyps; TSA, traditional serrated adenoma; SSA/P, sessile serrated adenoma/polyps; SD, standard deviation.


Figure 1. Representative results of FHIT and COX-2 immunostaining. (A) Negative FHIT immunostaining in SSA/P. (B) Positive FHIT immunostaining in traditional serrated adenocarinmas. (C) Negative COX-2 immunostaining in SSA/P. (D) Positive COX-2 immunostaining of a carcinomatous area of carcinoma in adenomas. Scale bar, $200 \mu \mathrm{m}$. Magnification, x200. FHIT, Fragile Histidine Triad; COX-2, cyclooxygenase 2; SSA/P, sessile serrated adenoma/polyp.

or Mann-Whitney $\mathrm{U}$ test. $\mathrm{P}<0.05$ was considered to indicate a statistically significant difference. All statistical calculations were performed using Stat Flex (version 6.0; Artech Co., Ltd., Osaka, Japan).

\section{Results}

Clinicopathological features. The clinicopathological features of the patients considered in the present study are summarized in Table I. All TSAs were localized in the distal region with the exception of one case. However, no significant differences were observed between HPs, TSAs, SSA/Ps, adenomas, CIAs and EPCs for any other clinicopathological feature. Flat elevated or depressed tumors were observed in 15/18 EPCs, whereas 11/20 HPs, 14/26 SSA/Ps, 12/20 adenomas, 11/20 CIAs and all TSAs had polypoid features. The submucosa was invaded in $6 / 20$ CIAs and 10/18 EPCs. Only 1 EPC demonstrated lymphatic invasion, while 2 CIAs and 3 EPCs exhibited venous invasion.

FHIT, COX-2, MLH1, MSH2 and P53 expression in early colorectal neoplasms. The results of the immunohistochemical staining experiments are summarized in Fig. 1 and 
Table II. Reduced or no FHIT expression was detected in $20 \%$ of HPs (30.8\% of MVHPs and 0\% of GCHPs), $50 \%$ of TSAs, $92.3 \%$ of SSA/Ps, $0 \%$ of non-serrated adenomas, $10 \%$ of CIAs and $44.4 \%$ of EPCs. Aberrant FHIT expression was frequently observed in TSAs $(\mathrm{P}<0.0003)$, SSA/Ps $(\mathrm{P}<0.0001)$ and EPCs $(\mathrm{P}<0.0007)$, but rarely occurred in non-serrated adenomas and CIAs. In addition, reduced or no FHIT expression was significantly more frequent in SSA/Ps (92.3\%) compared with in TSAs (50\%). COX-2 expression was observed in $60 \%$ of CIAs, $25 \%$ of non-serrated adenomas, $22.2 \%$ of EPCs and $3.2 \%$ of serrated polyps (5\% of HPs, $0 \%$ of TSAs and $3.8 \%$ of SSA/P). COX-2 expression was more common in non-serrated adenomas (25\%) compared with serrated polyps (3.2\%) $(\mathrm{P}=0.01)$. Furthermore, COX-2 expression was also more frequent in CIAs (60\%) compared with in EPCs $(22.2 \%, \mathrm{P}<0.05) .16 .7 \%$ of EPCs and $11.5 \%$ of SSA/Ps demonstrated a loss of MLH1/MSH2 expression. P53 overexpression was significantly increased in EPCs (77.8\%) and CIAs (60\%) compared with HPs (0\%), TSAs (6.6\%), SSA/Ps (0\%) and non-serrated adenomas (10\%) $(\mathrm{P}<0.01)$. The association between protein expression and clinicopathological data (tumor size, tumor location, macroscopic features, depth of invasion and lymphatic and venous invasion) was also analyzed. Absence of COX-2 expression was prevalent in FHIT-negative SSA/Ps compared with in FHIT-positive SSA/Ps ( $\mathrm{P}=0.08)$. No significant associations were observed between the expression of MLH1, MSH2 or P53 and other clinicopathological parameters.

\section{Discussion}

The ACS and the serrated pathway are two opposing models explaining the natural history of colorectal neoplasms $(3,28)$. Serrated colorectum polyps possess distinct histological features and malignant potential (3). CRCs arising from the serrated pathway are also often associated with CIMP (4) and MSI due to the methylation of $M L H 1$ (6-8). Previous data has revealed that MMR deficiency, dependent on MLH1 protein expression and CIMP-high state, is significantly associated with reduced FHIT expression in patients with CRC (14-17). COX-2 overexpression in CRC is inversely associated with MSI (20-22) and CIMP $(22,23)$. To clarify the molecular mechanism of serrated tumorigenesis, the expression of FHIT, COX-2, MLH1, MSH2 and P53 was examined in endoscopically-resected serrated and non-serrated colorectal neoplasias.

To the best of our knowledge, no study to date has compared FHIT expression levels in SSA/Ps, TSAs, non-serrated adenomas and adenocarcinomas. The present results indicated that reduced FHIT expression was frequent in serrated neoplasias, particularly SSA/Ps, when compared with non-serrated neoplasias. Aberrant crypt foci in the right side of the colon, one of the characteristics of MVHP, is a precursor lesion for the SSA/P-cancer sequence (29) and shows reduced FHIT expression $(13,30)$. In the present study, reduced or no FHIT expression was detected in 30.8\% of MVHPs. Methylation of the promoter region of FHIT has been reported in TSA, in particular in $13 \%$ of serrated adenomas with low-grade atypism, in $50 \%$ of serrated adenomas with high-grade atypism and in $33.3 \%$ of serrated adenomas with carcinomas (31). The reduction or loss of FHIT protein expression may be affected by extensive promoter methylation in CIMP-high CRC (17). 
These observations indicate that silencing of the FHIT expression performs an important role in the development of SSA/P and TSA and supports an association between the serrated pathway and a CIMP-high state in CRC development.

Previous studies have examined COX-2 expression in serrated polyps (32-35), demonstrating that HPs and SSA/Ps infrequently overexpress COX-2 compared with non-serrated adenomas and TSAs. In the present study, COX-2 overexpression was more common in non-serrated adenomas (25\%) compared with in serrated polyps, that is, HPs and SSA/Ps and TSAs (3.2\%, $\mathrm{P}=0.01)$. The present results demonstrated a comparatively lower percentage of COX-2 expression in SSA/Ps (3.8\%) and TSAs $(0 \%)$. The discrepancies between the present and previous studies may derive from differences in the interpretation of COX-2 immunostaining results and the antibodies used for the experiments. COX-2 overexpression in CRC is inversely associated with MSI (20-22) and CIMP status $(22,23)$. CIMP is associated with the serrated pathways, and Toyota et al (23) reported that COX-2 methylation is clearly associated with CIMP in colorectal tumors. Therefore, COX-2 overexpression may perform a secondary role in this pathway (32). In addition, cells not expressing COX-2 were common in FHIT-negative SSA/Ps, suggesting an association between the serrated pathway and CIMP-high CRC development.

Aberrant expression of FHIT and MLH1, and a lack of COX-2 expression in EPCs were frequent when compared with non-serrated adenomas and CIAs. The expression pattern of these proteins in EPCs partially matched that of serrated polyps. These results supported the hypothesis that the molecular pathogenesis of EPCs is distinct from that of ACS with respect to the expression of FHIT, MLH1 and COX-2. EPCs may therefore contain tumors derived from the serrated pathway as well as ACS.

MLH1/MSH2 silencing by CIMP is a critical molecular mechanism for SSA/P progression, resulting in cancers with MSI-high expression $(3,4)$. In addition, a loss of MLH1/MSH2 immunohistochemical signals in the carcinogenesis of SSA/P has been reported previously (6-8). In the present study, three cases of SSA/Ps and three cases of EPC demonstrated a partial or total loss of MLH1/MSH2 protein. In addition, P53-positive rates were much higher in CIAs and EPCs compared with non-serrated adenomas. At the same time, P53-positive rates were rare in serrated polyps. P53 overexpression appears to become more frequent as tumors progress to higher grades of neoplasia, in agreement with previous studies $(36,37)$.

However, the present study has limitations. The number of female patients was reduced $(n=30)$ compared with the male counterparts $(n=90)$. Therefore, the present findings may not be sufficient for female patients. A larger study population is necessary to address this problem. Secondly, the information for several well-known biomarkers, including $\beta$-catenin and K-ras on colorectal carcinogenesis was not available in the present study. Additional examinations of these biomarkers may contribute to additional understanding of serrated tumorigenesis.

In conclusion, the present results indicated that aberrant expression of FHIT and the absence of COX-2 expression are associated with serrated tumorigenesis. In addition, EPC may contain tumors derived from the serrated pathway and ACS. The present findings of using biomarkers to distinguish the pathological features may be of a great importance for individualized medicine. The association between the expression of FHIT and COX-2 and the origin of adenocarcinoma in serrated polyps remains to be addressed.

\section{References}

1. Siegel R, Ma J, Zou Z and Jemal A: Cancer Statistics, 2014. CA Cancer J Clin 64: 9-29, 2014.

2. Vogelstein B, Fearon ER, Hamilton SR, Kern SE, Preisinger AC, Leppert M, Nakamura Y, White R, Smits AM and Bos JL: Genetic alterations during colorectal-tumor development. N Engl J Med 319: 525-532, 1988.

3. Mäkinen MJ, George SM, Jernvall P, Mäkelä J, Vihko P and Karttunen TJ: Colorectal carcinoma associated with serrated adenoma-prevalence, histological features, and prognosis. J Pathol 193: 286-294, 2001.

4. O'Brien MJ, Yang S, Huang CS, Shepherd C, Cerda S and Farraye FA: The serrated polyp pathway to colorectal carcinoma. Diagn Histopathol 14: 78-93, 2008.

5. Snover DC, Ahnen DJ and Burt RW: Serrated polyps of the colon and rectum and serrated polyposis. In: Bosman FT, Carneiro F, Hruban RH, Theise ND (eds). WHO classification of tumours of the digestive system. IARC P, Lyon, France, pp160-165, 2010.

6. Huang CS, Farraye FA, Yang S and O'Brien MJ: The clinical significance of serrated polyps. Am J Gastroenterol 106: 229-240, 2011.

7. Bettington M, Walker N, Clouston A, Brown I, Leggett B and Whitehall V: The serrated pathway to colorectal carcinoma: Current concepts and challenges. Histopathology 62: 367-386, 2013.

8. Cunningham JM, Christensen ER, Tester DJ, Kim CY, Roche PC Burgart LJ and Thibodeau SN: Hypermethylation of the hMLH1 promoter in colon cancer with microsatellite instability. Cancer Res 58: 3455-3460, 1998.

9. Ohta M, Inoue H, Cotticelli MG, Kastury K, Baffa R, Palazzo J, Siprashvili Z, Mori M, McCue P, Druck T, et al: The FHIT gene, spanning the chromosome 3p14.2 fragile site and renal carcinomaassociated $\mathrm{t}(3 ; 8)$ breakpoint, is abnormal in digestive tract cancers. Cell 84: 587-597, 1996.

10. Huebner K and Croce CM: Cancer and the FRA3B/FHIT fragile locus: It's a HIT. Br J Cancer 88: 1501-1506, 2003.

11. Kuwai T, Tanaka S, Kaio E, Hiyama T, Ito M, Kitadai Y, Sumii M, Yoshihara M, Haruma K and Chayama K: Clinical significance of Fhit expression in development of colorectal carcinoma of various macroscopic types. Int J Mol Med 12: 437-442, 2003.

12. Mimori K, Ishii H, Nagahara H, Sudo T, Yamashita K, Inoue H, Barnard GF and Mori M: FHIT is up-regulated by inflammatory stimuli and inhibits prostaglandin E2-mediated cancer progression. Cancer Res 66: 2683-2690, 2006.

13. Hao XP, Willis JE, Pretlow TG, Rao JS, MacLennan GT, Talbot IC and Pretlow TP: Loss of fragile histidine triad expression in colorectal carcinomas and premalignant lesions. Cancer Res 60: 18-21, 2000

14. Mori M, Mimori K, Masuda T, Yoshinaga K, Yamashita K, Matsuyama A and Inoue $\mathrm{H}$ : Absence of Msh2 protein expression is associated with alteration in the FHIT locus and Fhit protein expression in colorectal carcinoma. Cancer Res 61: 7379-7382, 2001.

15. Andachi H, Yashima K, Koda M, Kawaguchi K, Kitamura A, Hosoda A, Kishimoto Y, Shiota G, Ito H, Makino M, et al: Reduced Fhit expression is associated with mismatch repair deficiency in human advanced colorectal carcinoma. Br J Cancer 87: 441-445, 2002

16. Sarli L, Bottarelli L, Azzoni C, Campanini N, Di Cola G, Bader G, Iusco D, Salvemini C, Caruso G, Donadei E, et al: Abnormal Fhit protein expression and high frequency of microsatellite instability in sporadic colorectal cancer. Eur J Cancer 40: 1581-1588, 2004.

17. Al-Temaimi RA, Jacob S, Al-Ali W, Thomas DA and Al-Mulla F: Reduced FHIT expression is associated with mismatch repair deficient and high $\mathrm{CpG}$ island methylator phenotype colorectal cancer. J Histochem Cytochem 61: 627-638, 2013.

18. Brown JR and DuBois RN: COX-2: A molecular target for colorectal cancer prevention. J Clin Oncol 23: 2840-2855, 2005.

19. Sheehan KM, O'Connell F, O'Grady A, Conroy RM, Leader MB, Byrne MF, Murray FE and Kay EW: The relationship between cyclooxygenase-2 expression and characteristics of malignant transformation in human colorectal adenomas. Eur J Gastroenterol Hepatol 16: 619-625, 2004. 
20. Sinicrope FA, Lemoine M, Xi L, Lynch PM, Cleary KR, Shen Y and Frazier ML: Reduced expression of cyclooxygenase 2 proteins in hereditary nonpolyposis colorectal cancers relative to sporadic cancers. Gastroenterology 117: 350-358, 1999.

21. Karnes WE Jr, Shattuck-Brandt R, Burgart LJ, DuBois RN, Tester DJ, Cunningham JM, Kim CY, McDonnell SK, Schaid DJ and Thibodeau SN: Reduced COX-2 protein in colorectal cancer with defective mismatch repair. Cancer Res 58: 5473-5477, 1998

22. Ogino S, Brahmandam M, Kawasaki T, Kirkner GJ, Loda M and Fuchs CS: Combined analysis of COX-2 and p53 expressions reveals synergistic inverse correlations with microsatellite instability and $\mathrm{CpG}$ island methylator phenotype in colorectal cancer. Neoplasia 8: 458-464, 2006.

23. Toyota M, Shen L, Ohe-Toyota M, Hamilton SR, Sinicrope FA and Issa JP: Aberrant methylation of the Cyclooxygenase $2 \mathrm{CpG}$ island in colorectal tumors. Cancer Res 60: 4044-4048, 2000.

24. Japanese General Rules for Clinical and Pathological Studies on Cancer of the Colon, Rectum and Anus. Japanese Society for Cancer of the Colon and Rectum, 2009.

25. Schlemper RJ, Riddell RH, Kato Y, Borchard F, Cooper HS Dawsey SM, Dixon MF, Fenoglio-Preiser CM, Fléjou JF, Geboes K, et al: The Vienna classification of gastrointestinal epithelial neoplasia. Gut 47: 251-255, 2000.

26. Saitoh Y, Waxman I, West AB, Popnikolov NK, Gatalica Z, Watar J, Obara T, Kohgo Y and Pasricha PJ: Prevalence and distinctive biologic features of flat colorectal adenomas in a North American polulation. Gastroenterology 120: 1657-1665, 2001.

27. Beak MJ, Kang H, Kim SE, Park JH, Lee JS, Paik YK and Kim H: Expression of hMLH1 is inactivated in the gastric adenomas with enhanced microsatellite instability. Br J Cancer 85: 1147-1152, 2001.

28. Jass JR: Classification of colorectal cancer based on correlation of clinical, morphological and molecular features. Histopathology 50: 113-130, 2007.

29. Inoue A, Okamoto K, Fujino Y, Nakagawa T, Muguruma N, Sannomiya K, Mitsui Y, Takaoka T, Kitamura S, Miyamoto $\mathrm{H}$, et al: B-RAF mutation and accumulated gene methylation in aberrant crypt foci (ACF), sessile serrated adenoma/polyp (SSA/P) and cancer in SSA/P. Br J Cancer 112: 403-412, 2015
30. Vaiphei K, Rangan A and Singh R: Aberrant crypt focus and fragile histidine triad protein in sporadic colorectal carcinoma. World J Gastrointest Oncol 4: 250-258, 2012.

31. Dong SM, Lee EJ, Jeon ES, Park CK and Kim KM: Progressive methylation during the serrated neoplasia pathway of the colorectum. Mod Pathol 18: 170-178, 2005.

32. Kawasaki T, Nosho K, Ohnishi M, Suemoto Y, Glickman JN, Chan AT, Kirkner GJ, Mino-Kenudson M, Fuchs CS and Ogino S: Cyclooxygenase-2 overexpression is common in serrated and non-serrated colorectal adenoma, but uncommon in hyperplastic polyp and sessile serrated polyp/adenoma. BMC Cancer 8: 33, 2008.

33. Takeuchi M, Kobayashi M, Ajioka Y, Honma T, Suzuki Y, Azumaya M, Narisawa R, Hayashi S and Asakura H: Comparison of cyclo-oxygenase 2 expression in colorectal serrated adenomas to expression in tubular adenomas and hyperplastic polyps. Int J Colorectal Dis 17: 144-149, 2002.

34. Arao J, Sano Y, Fujii T, Kato S, Fu KI, Yoshino T, Ochiai A, Fujimori T and Yoshida S: Cyclooxygenase-2 is overexpressed in serrated adenoma of the colorectum. Dis Colon Rectum 44: 1319-1323, 2001

35. Maekawa M, Sugano K, Sano H, Miyazaki S, Ushiama M, Fujita S, Gotoda T, Yokota T, Ohkura H, Kakizoe T and Sekiya T: Increased expression of cyclooxygenase-2 to -1 in human colorectal cancers and adenomas, but not in hyperplastic polyps. Jpn J Clin Oncol 28: 421-426, 1998.

36. Castells A, Payá A, Alenda C, Rodríguez-Moranta F, Agrelo R, Andreu M, Piñol V, Castellví-Bel S, Jover R, Llor X, et al: Cyclooxygenase 2 expression in colorectal cancer with DNA mismatch repair deficiency. Clin Cancer Res 12: 1686-1692, 2006.

37. Wasa R, Matsukuma S, Abe H, Kuwabara N, Suda K, Arakawwa A and Kitamura S: Histopathological studies of superficial-type early colorectal carcinoma. Cancer 77: 44-50, 1996. 